String theory: results, magic and doubts

This content has been downloaded from IOPscience. Please scroll down to see the full text. 2013 Phys. Scr. 2013014026

(http://iopscience.iop.org/1402-4896/2013/T158/014026)

View the table of contents for this issue, or go to the journal homepage for more

Download details:

IP Address: 137.138.125.164

This content was downloaded on 19/05/2014 at 12:54

Please note that terms and conditions apply. 


\title{
String theory: results, magic and doubts
}

\section{Eliezer Rabinovici}

Racah Institute of Physics, Hebrew University of Jerusalem, Israel

and

CERN, Geneva, Switzerland

E-mail: eliezer@vms.huji.ac.il

Received 1 October 2013

Accepted for publication 4 December 2013

Published 27 December 2013

Online at stacks.iop.org/PhysScr/T158/014026

\begin{abstract}
This talk was given at a special place and on a special occasion for a special audience. It describes the results, magic and doubts that an attempt to construct a theory based on the idea that the basic constituents of matter can also be extended objects has led to. The emphasis is on the spirit behind the ideas.

PACS numbers: 11.10.-z, 11.15.-q, 11.25.-W, 11.27.+d, 11.30.-j, 11.40.-q, 11.55.-M, 11.80.-M
\end{abstract}

\section{Shortly Before arriving in Stockholm, \\ Klein received a letter from his good friend Pauli which reads in part:}

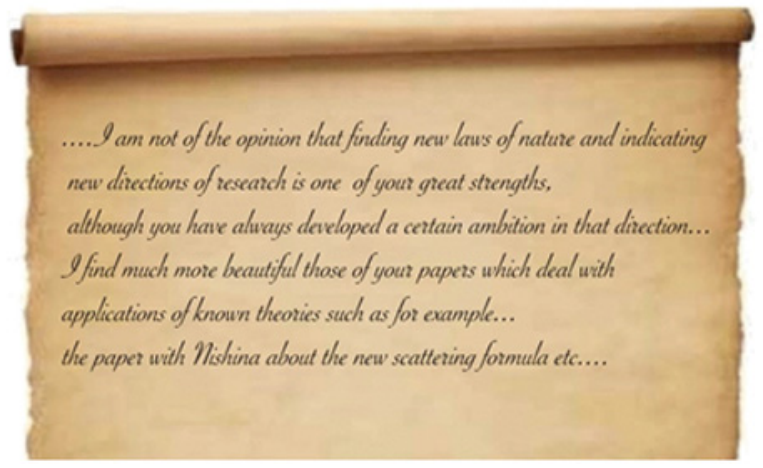

Figure 1. A letter between friends. Try to imagine all the e-mails of this nature sent between 'friends' that one will never get to see. Credit: Pauli Archive at CERN.

My first acquaintance with Sweden was when I served at the age of 17 as a deck boy on the Swedish ship 'Vikingland'. After several weeks the captain decided that perhaps I could be of more use in the navigating room and even trusted the helm of the ship to my hands.

Let me now go further back in time to the early decades of the 20th century when a real navigator in the true Viking tradition, a professor named Klein was given a position in Sweden. He and another physicist, Kaluza, came up with an extremely original idea on how to unify the two known forces at the time: gravity and electromagnetism. To do this Klein made a very bold jump: he considered the possibility

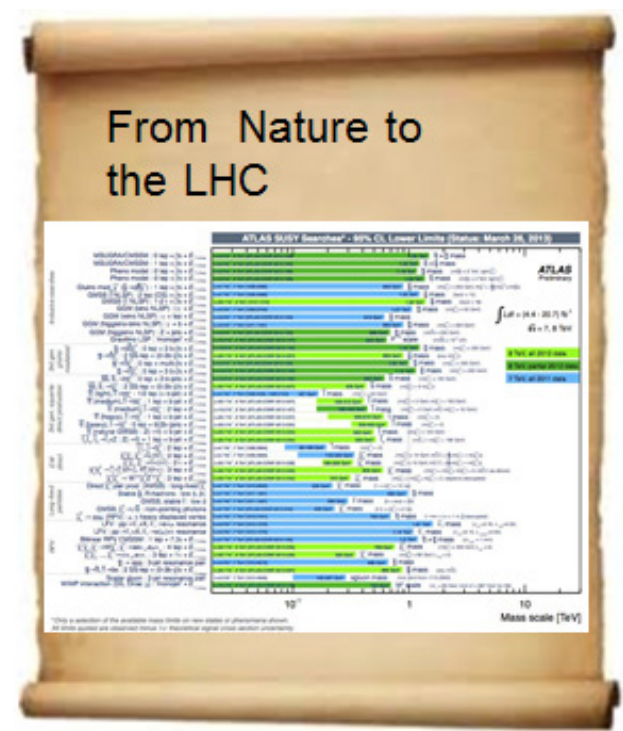

Figure 2. Although you cannot discern the full content of the message in this figure, its impact is rather devastating. It states that, at the time of the meeting for the Nobel symposium, the experiments have not found any new particles, beyond the Higgs particle. It does, however, contain quantitative information; it states that the mass of such new particles if they indeed exist cannot be lower than a certain value, called the lower bound. Credit: ATLAS@CERN.

that our world has one extra dimension. He assumed that dimension to have a non-trivial topology: that of a circle of very small radius so as to make the theory consistent with the fact that such an extra dimension had not been observed experimentally. A single force, gravity, existing in five dimensions would manifest itself to a low energy physicist as 


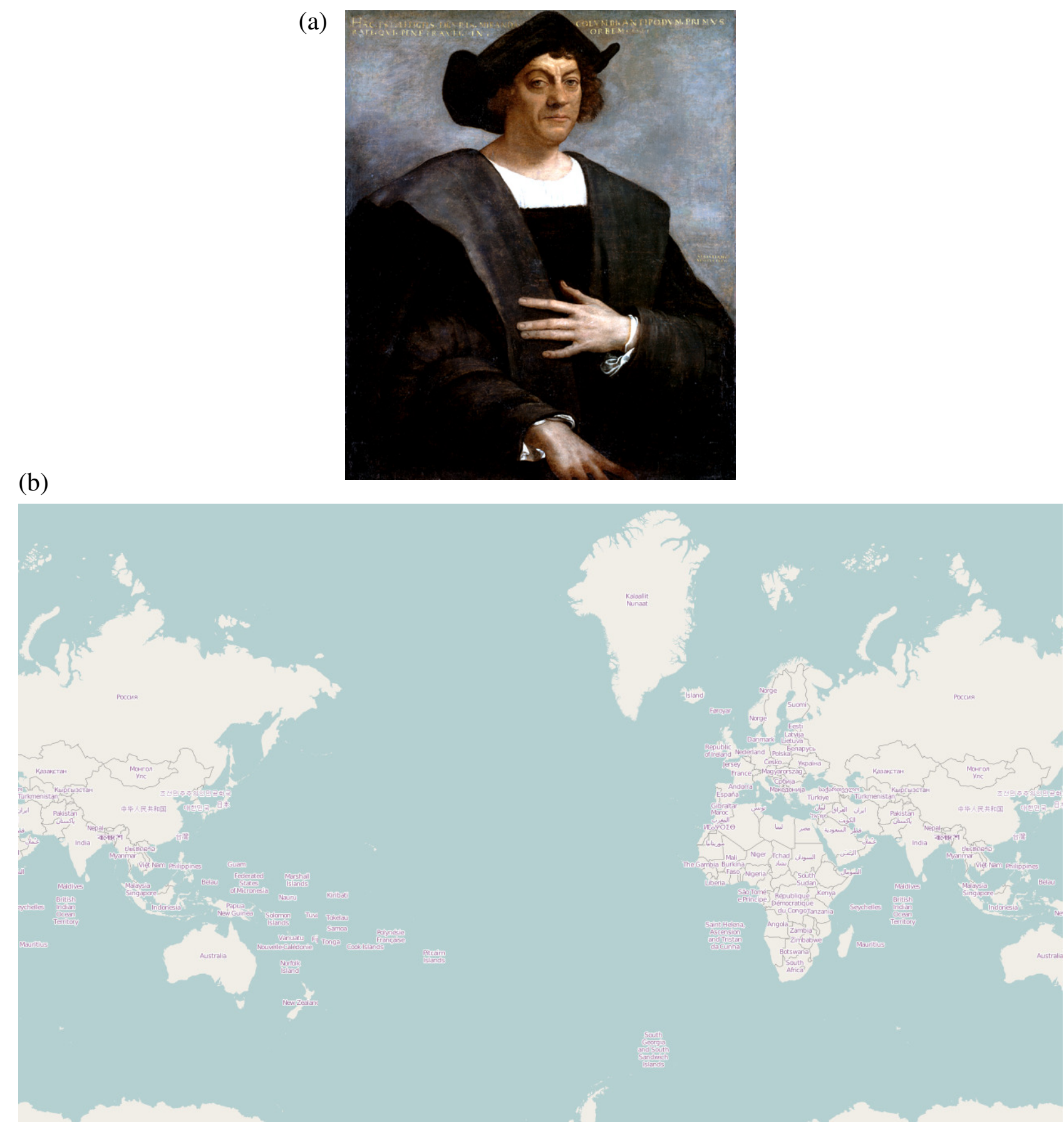

Figure 3. (a) Christopher Columbus; (b) on a different planet, the journey of Columbus could have ended in a China or India on that other planet. Credit: (b) @ OpenStreetMap contributers. CC BY-SA.

a four-dimensional theory of gravity, electromagnetism and an extra scalar.

Klein had a friend called W Pauli who was 6 years younger than him. According to A Pais, Klein's navigation efforts led Pauli to write the letter, given in figure 1, to Klein. Imagine yourself having as brilliant an idea as that of Kaluza and Klein and then getting such a non-supportive letter from your supposedly dear friends!

Well, string theorists also have the Viking navigator component in them, and next take a look at the letter that we, the particle physics community have received-not from our theorist friends, but from Nature, through our experimental colleagues (figure 2).
The experimentalists announced lower bound after lower bound for the masses of each of the fantasy particles the theoreticians had envisaged. The fantasy particles ranged from the masses of composite quarks to those of the supersymmetrical partners of the known particles. But as older navigators, we will not be deterred. We will patiently wait for the completion of the analysis of the presently gathered data and more results from the various upgrades of the Large Hadron Collider.

We are deeply appreciative of the outstanding achievement of our experimental colleagues. Against all odds they have found a new particle. Its properties will be analysed in great detail, with a view to finding something new. 


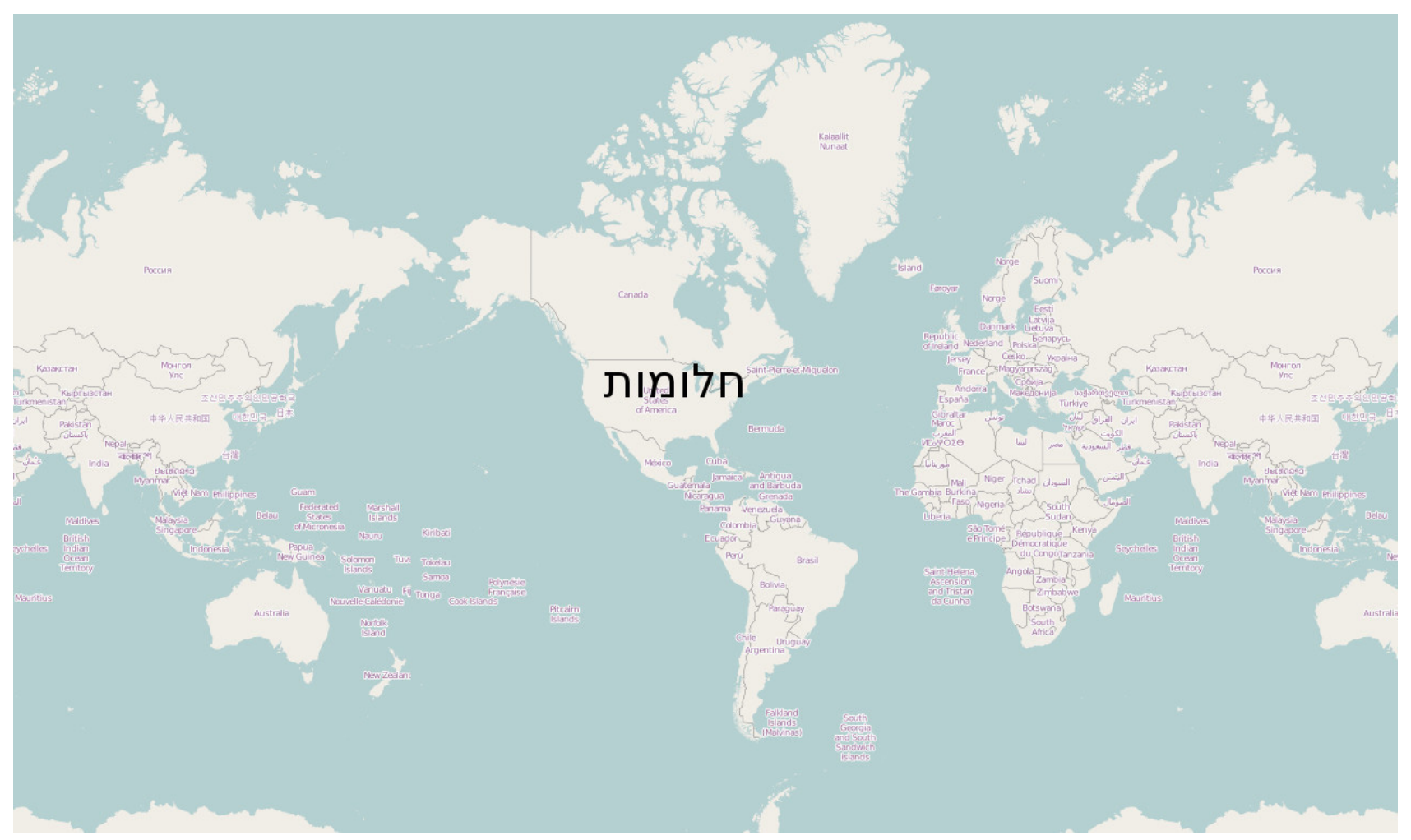

Figure 4. As the text in the figure indicates, there are many dreams in our field that need to wait for a discovery. Columbus reached America; in contrast, we, physicists, are still waiting for our America. Credit: ๑ OpenStreetMap contributers. CC BY-SA.

For me, the earlier discovery at CERN in 1982/3 of the massive spin 1 particles that carry the weak interactions validated the BEH (Brout, Englert, Higgs) mechanism for the non-zero mass generation. In a sense, three scalar particles had already been discovered. The issue of whether a fourth one would be discovered was very important. Without a fourth scalar particle, the situation would be equivalent to that of Columbus reaching the Orient directly without reaching America, thereby validating his theory that the world is spherical (figure 3).

Many theorists would have been delighted if it had turned out that there was no evidence for a fundamental (at least at these scales) scalar particle. That would have taught us that the three particles responsible for the Ws and $\mathrm{Z}$ obtaining masses were actually composite. These imposters, namely, objects masquerading as elementary particles would have taught us that new physics beyond the Standard Model would certainly have been within reach. In addition theorists had many other fantasies that they wished to explore, such as extra dimensions and supersymmetrical particles. Funding agencies, however, wished for a clear and present day discovery that would steal the headlines of the newspapers and make the breaking news, thereby convincing the public and the politicians that their, actually rather modest investment on any relative scale, is justified. Funding matters have become (too) dominant in our curiosity driven research. Anyhow, the funding agencies' wishes were granted: the 'Americas' have yet to be reached on our trip (figure 4).

Each generation wants to have its share of new insights and new discoveries. But let us stop moaning for a moment and celebrate the Standard Model, which was built under the assumption that the basic constituents of nature are point particles. It seems that some humans over the ages (known nowadays as theoretical particle physicists) have ignored the NOH principle repeatedly; that is, Nobody Owes Humanity. We have no right to expect that just a few terse accessible slides in a talk should cover all of the known basic constituents of matter and the laws governing them. And yet, at this stage, they do!

The affirmation of the success of the Standard Model does ignore the decades' old challenge presented by the astrophysical observation of what is called dark matter. The essentially vanishing magnitude of the dark energy manifests, I believe, the presence of an underlying scale and conformal invariance in nature.

So here, in figure 5, are all known particles.

All of these known particles are assumed to be described by basic constituents that are point particles. As they move in time they span a world-line whose character and significance are determined by the forces they experience and the laws of quantum mechanics (figure 6).

The equations displayed in figure 7 are not only rather compact, in addition, they allow us to extract out numbers that can be used to predict the results of experiments: predictions that have been verified. Let us concentrate on the best cases. Some electromagnetic properties of the electron and muon, such as g-2 can be predicted up to 11 digits beyond the 0 .

That prediction has been verified. I know of no other human endeavour that has reached such accuracy. For these predictions, one needs only two numbers: the mass and the charge of the lepton.

Imagine, for example, you were to ask a person from the life sciences to request two numbers from nature and then calculate something with such accuracy, say the life-span of 


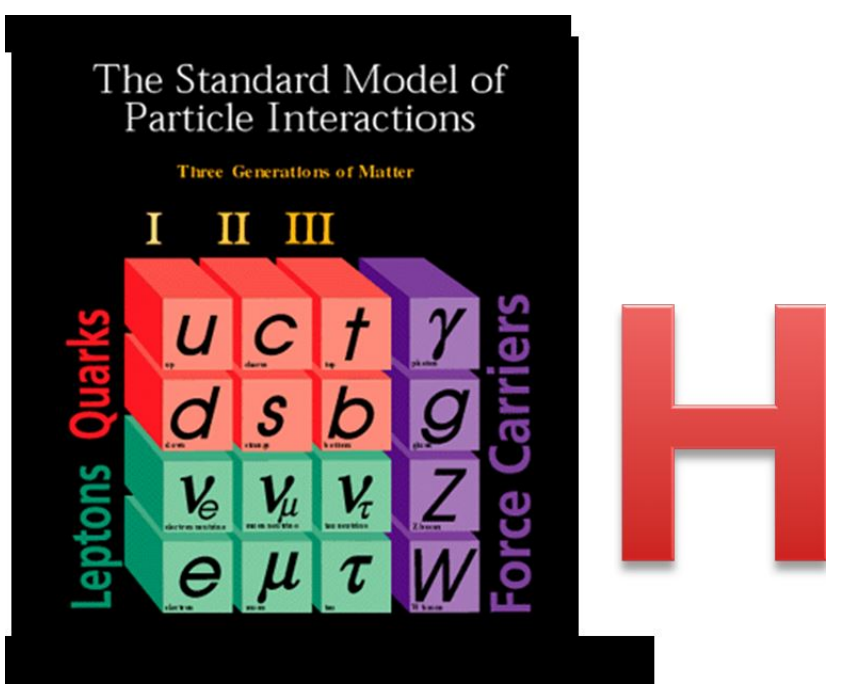

Figure 5. All of the present suspects of being the elementary constituents fit on one slide. Credit: Fermilab.

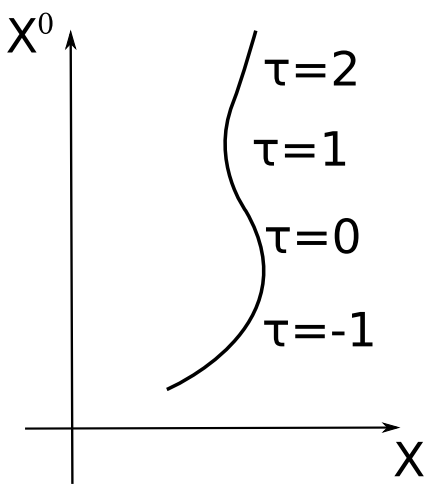

Figure 6. A point particle traces a world-line as it propagates in time. The vertical axis represents the time $X^{0}$ and the horizontal axis represents the position of the particle.

$$
\mathscr{L}_{0}^{\prime}=\bar{\psi} i \gamma^{\mu}\left(\partial_{\mu}+i e A_{\mu}\right) \psi-m \bar{\psi} \psi
$$

$$
\mathscr{L}=\left(\mathrm{D}_{\mu} \phi\right)^{\dagger}\left(\mathrm{D}^{\mu} \phi\right)+\mu^{2} \phi^{\dagger} \phi-\lambda\left(\phi^{\dagger} \phi\right)^{2}-\frac{1}{4} F_{\mu v} F^{\mu v}
$$

Figure 7. Up to the laws governing gravity and missing indices, these are the laws that govern matter as we know it today. Note that they fit on one slide — or in one figure!

some species. They would look horrified because this cannot be done.

For the weak interactions, the best predictions are of the order of $0.1 \%$, and for the coloured forces there are properties that can be calculated to a $1 \%$ level. All in all, amazing, and yet we are not satisfied (figure 8).

After these amazing successes, why try to change such a winning team? Why try to go beyond a point particle based theory to explain the fundamental constituents of nature? One could argue that the nagging issue of trying to have a well-defined quantum theory of gravity was the cause, but in fact the historical route was through the experimental results

\section{The level of professionalism}

\section{- In calculations involving the electromagnetic force 0.00115965218 \\ - In calculations involving the weak force, $0.1 \%$ \\ - In calculations involving the color force $1 \%$}

Figure 8. The amazing agreement between theory and experiment. The best cases have been chosen.

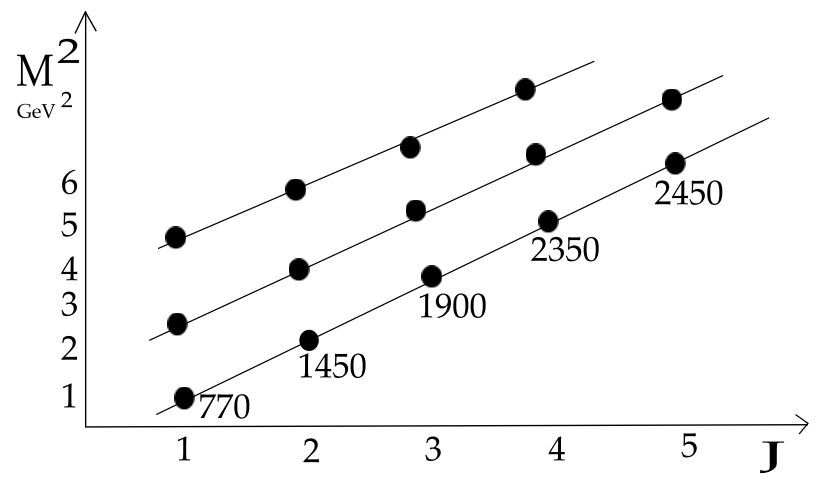

Figure 9. The experimental discovery of hadrons fitting on Regge trajectories was one source of inspiration leading to the first attempts to construct a string theory. String theory first appeared to describe strong interactions. Only later was it realized that the correct theory of quark and hadrons is quantum chromodynamics... and that string theory is a theory of quantum gravity.

in hadronic physics. Many new hadrons were found, and they seemed to fall neatly along the theorist's Regge trajectories. They proliferated, while no hadronic subconstituents were detected (figure 9).

Mechanistic models producing such Regge trajectories of particles led to the idea of considering the particles as being the vibrations of strings. Once the strings were there, being considered to be part of the story, one thing led to another. The moment one relinquishes the extreme view that elementary particles must be based on point particles, new horizons open. Imagine for a moment the theorists and experimentalists in the world of painting. What is the basic block of a painting? Dots? Brush strokes? Islands of colour? From far away, that may look irrelevant, but actually the details are seen at short distances and the global structure they convey can be apparent even from a distance (figures 10-12).

Let us examine point particles and strings.

When the world-lines of particles collide, their interactions are harsh and require renormalization. Moreover, when gravitational interactions are at play, generically, they are even too strong to handle in a calculable manner.

One of the unexpected results of expanding the picture to look at sheets instead of lines was that, when the world-sheets spanned by strings in string theory collide, their interactions are much softer than those of the world-lines. There are strong indications that, even when gravitational forces are involved, there is no need for an infinity of renormalizations. This allows the theory to be useful (figure 13).

Moreover, in the limit when the string tension vanishes, the low energy degrees of the open string system lead to 


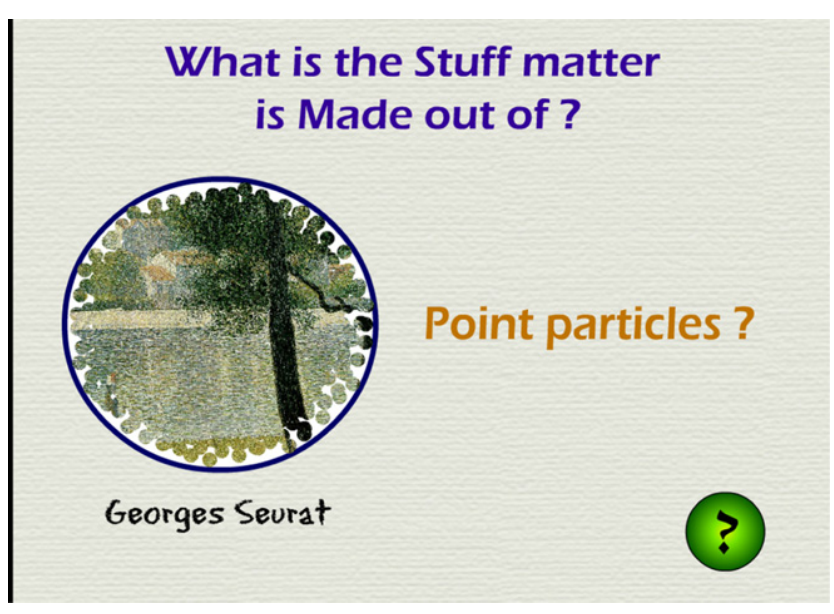

Figure 10. A painting built out of point particles, where each point is one dot of colour, and the dots are the constituents that comprise the image as a whole.

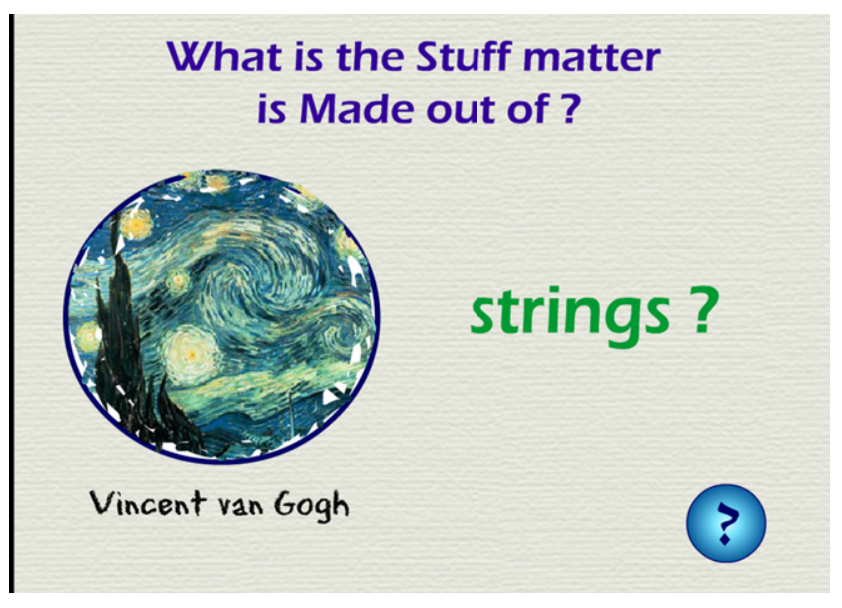

Figure 11. A painting whose basic constituents are strings, comprised of painterly brush strokes.

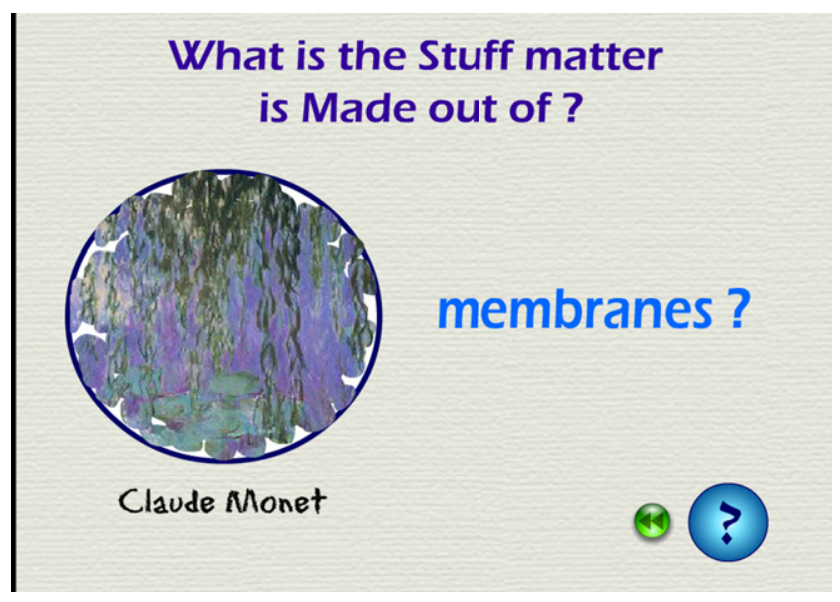

Figure 12. A painting whose constituents are two-dimensional patterns, effectively membranes.

the field theoretical Yang-Mills theory. For closed strings the gravitational theory of Einstein emerges. Another old Nordic initiation procedure is respected: Bohr's correspondence principle (figure 14).
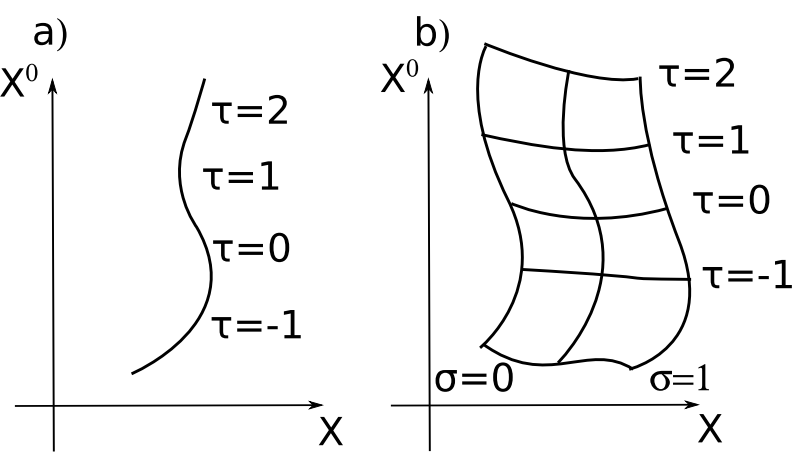

a)

b)
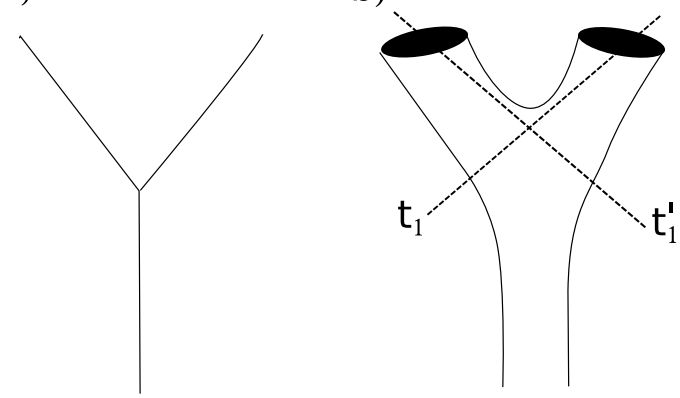

Figure 13. The upper part shows (a) a world-line spanned by a propagating particle and (b) a world-sheet spanned by a propagating string. The lower part shows their possible respective interactions. Those of strings (b) are less harsh.

\section{Open string}

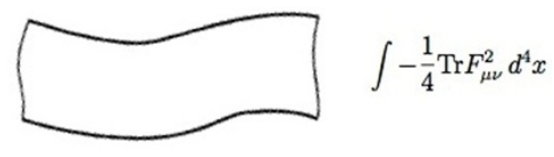

Closed string

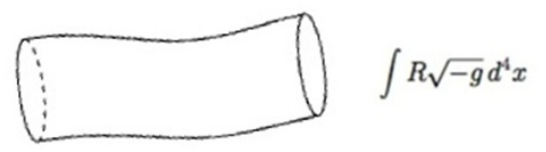

Figure 14. Bohr's correspondence principle in action. Opened and closed string systems reduce to Yang-Mills and gravity theories.

The significance of scientific research is not only measured by the quality of its results, but also by the quality of the questions it provokes.

Point particles led to the 'classical' 'why?' questions: Why are there three generations? Why do they have the properties they have? With strings, new questions of a somewhat different flavour have been added (figure 15).

So we ask: Why? Why? Why?

- Why does a universe have four dimensions?

- Why is there only one universe?

- Why does string theory have magic?

In seeking to address some of these questions, I will deal here with a couple of the roles that string theory has played over the years.

It has a role as an innovator: new unexpected properties have been discovered. String theory also has a role as a 


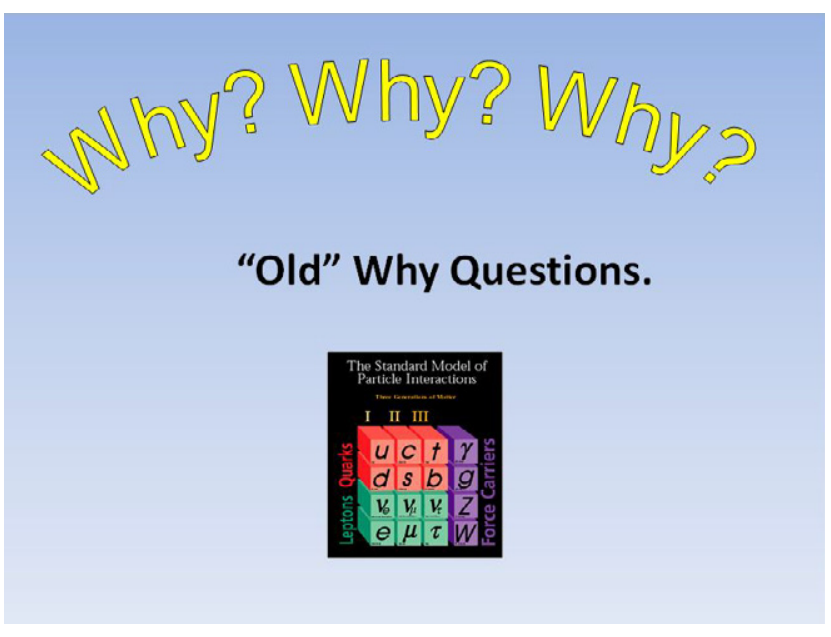

Figure 15. An old 'why?' question was, why did nature need a seeming redundance including three generations of very similar particles? Credit: Fermilab.

vindicator: it emulated many interesting features that certain field theories possess; time and again string theory has been a place where innovative ideas that failed to be proven elsewhere were actually vindicated. I will consider these roles in greater detail.

String theory as an innovator has turned the query what the 'dimension' of space-time must be into a scientific question: string theories seem to need a certain number, called the Virasoro central charge, to equal 26 and/or 15.

There are cases when these numbers can be translated to mean that the number of dimensions of space-time has to be either 26 or 10 , in order that strings may consistently propagate. Kaluza and Klein had already suggested extra dimensions for the purpose of unification, but for them, the number of dimensions was obtained from the bottom up. For the strings it was obtained from top to bottom and was a necessary condition. The ten-dimensional case is stable, thanks to the invention of a property called supersymmetry. This was the property of the matter on the two-dimensional world-sheet that represents the string propagating in space-time.

String theory has infinitely more particles than a regular point particle theory. These states are very massive and one of their roles is to make this theory containing gravity a finite, well-defined theory. Such a theory has been the holy grail of theoretical physics for many years.

String theory also has magic, which casts its spell. One such example of the magic is that when the theory is built out of strings there is no concept in mathematics that remains unambiguous. Symmetries, sometimes called dualities, identify objects which, when probed by point particles, are usually considered as different from each other.

There are examples for each of these cases. One familiar notion is related to the metric.

Metric. With extended objects there are cases where large objects and small ones give rise to exactly the same $S$ matrix elements, for example, when one extra dimension is a compact
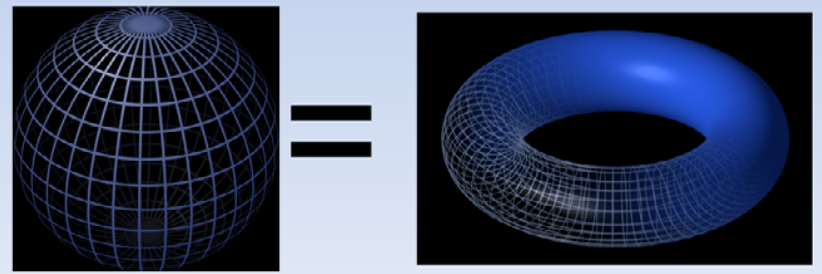

Figure 16. There are cases when a string probe cannot distinguish a sphere from a torus though their topologies are different.

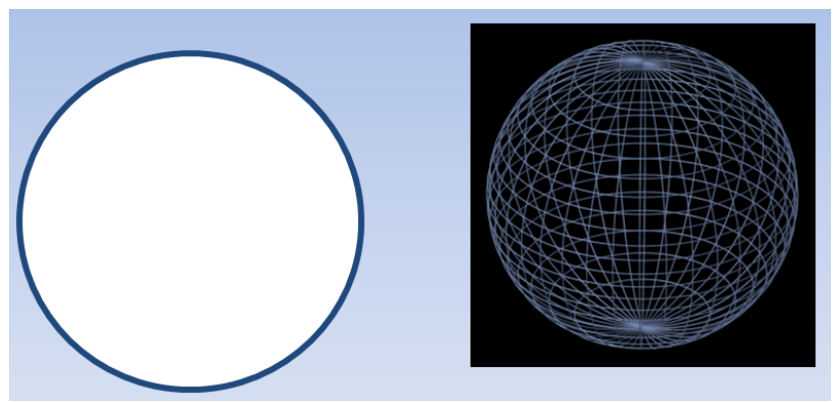

Figure 17. In some cases a string identifies spaces with different dimensions.

circle of the Kaluza-Klein type. A string propagating in a background containing a circle of radius $R$ (which could be large) would measure the same physics as a string moving in a background of radius $1 / R$ in appropriate units.

Large $=$ small .

$$
R \leftrightarrow \frac{1}{R}
$$

This type of symmetry, called $T$ duality, can be related to a residual gauge symmetry following a BEH mechanism in some cases in which, for example,

$$
S U(2) \times S U(2) \rightarrow U(1) \times U(1) .
$$

Another mathematical concept is topology.

Topology. A string will in some cases identify different topologies.

There are cases where there is a symmetry which identifies a surface of a two-dimensional sphere with a torus (figure 16).

Even the number of dimensions is not immune from ambiguities.

Dimension. For string-scale objects, group manifolds for example are identified with their Cartan sub torus. The former has as its dimension the dimension of the group, the latter the rank of the group, which is almost always smaller and rarely equal to the dimension. For the group manifold $S U(2)$, the dimension is three and the rank one. Thus, in some cases as far as dimensions are concerned $3=1$.

$S U(2)$ has $\operatorname{dim}=3$, its rank $=1$ (figure 17).

In other cases such as for the AdS/CFT correspondence, the same object can be described by a four-dimensional quantum field theory or a ten-dimensional string theory. 


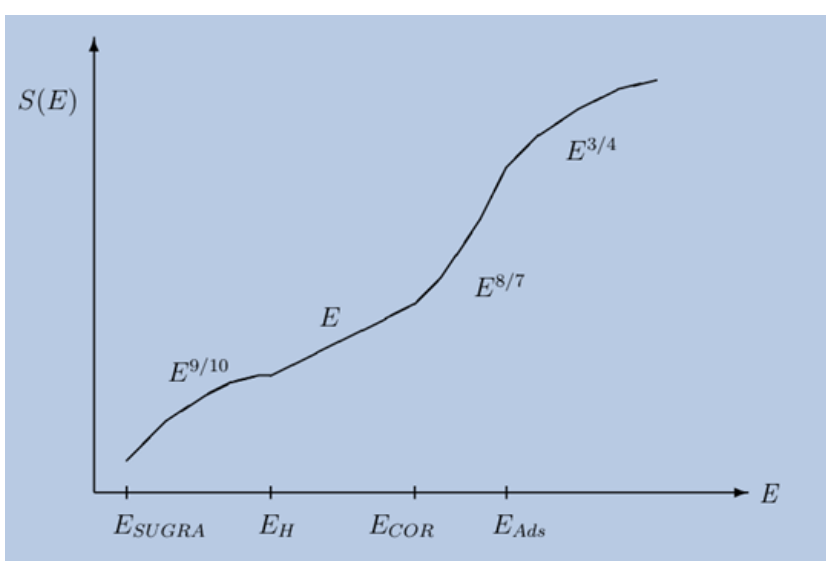

Figure 18. A string theory identified with a field theory exhibits, at different energy scales, different dependence of the entropy on the energy of the system. This reflects different effective dimensions. Credit: among others J L F Barbon and E Rabinovici.

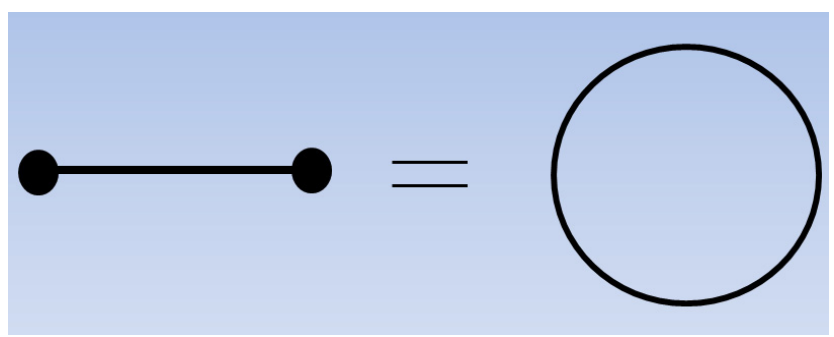

Figure 19. A case in which a string probe identifies a circle with a closed line segment containing two singular points at its edges.

$4=10$, in this instance. An analysis can refine this to state that, in the infrared, the system is ten dimensional, whereas at the ultraviolet it is only four dimensional (figure 18).

Imagine that our world as we know it now is the infrared of such a theory, which has a smaller UV dimension. Such a situation could make gravity a much kinder theory.

Next, consider time-like singularities.

Singularities. With extended objects, a circle of a certain radius, a very smooth object, becomes equivalent to an orbicircle (i.e., a circle with a certain identification) of some radius. The latter has singularities at its edges (figure 19).

One can continue to escalate the nature of the things one can identify. There are cases when a system can be described as living on a commutative manifold by one set of degrees of freedom while, with another set, it would be described as living on a non-commutative manifold (figure 20).

Commutativity. There are recent examples where, in the presence of fluxes, even associativity becomes ambiguous. To conclude, dualities with extended objects as probes relate cases of different: geometry, topology, number of dimensions, singularity structure, non-commutativity and associativity properties.

Recently, we have also added cases where, when described in one manner, the universe undergoes a big crunch and time, like when the son of Chronos is gobbled up, describes an eternal universe in some other manner (figure 21).

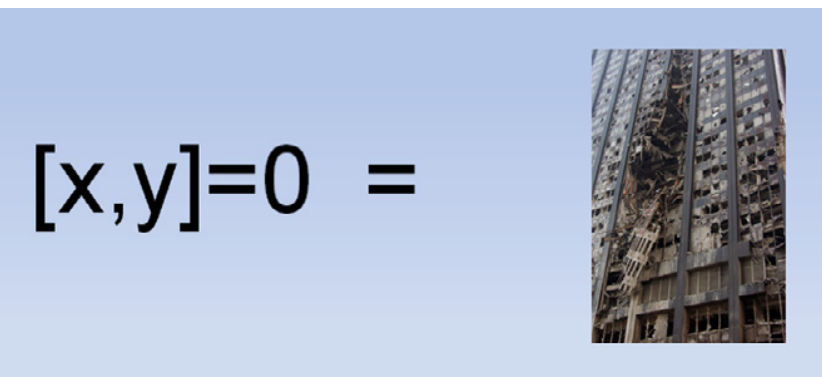

Figure 20. A commutative and a non-commutative manifold were identified by string probes. Credit: FEMA/Michael Rieger.

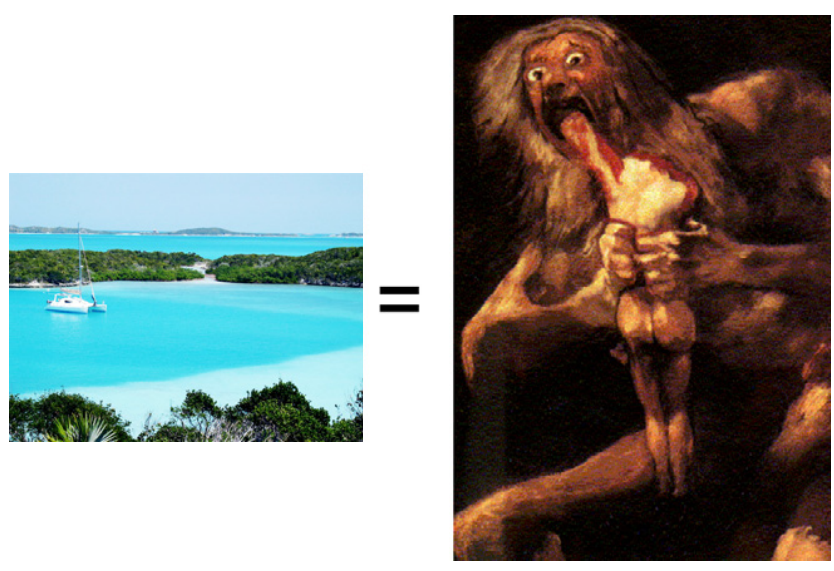

Figure 21. There are cases where strings identify a space of infinite extent in time with one which comes to an abrupt end/crunch. Credit: (left) @) Ryan Kozie. CC BY; (right) Goya Museo.

Usually singularities are a reflection of a breakdown of an approximation. However, it turned out that by a complementarity/duality argument, a system containing certain big crunches can be described just as well by a system which lives forever, totally ignorant of the crunch.

Research in string theory has led to many unexpected connections.

Complex problems in heavy ion scattering, strongly coupled condensed matter systems and hydrodynamical fluids have been related usefully to problems in general relativity including problems in black hole physics.

Less surprising in principle, but surprising by their impact are relations uncovered between string theory problems and frontline problems in mathematics.

String theory also shows itself as an emulator and vindicator. Studies in field theory before and after the period following the 1985 developments in string theory have led to many educated conjectures about dynamical and symmetry-related properties of various strongly coupled systems.

Examples of this are proving the role of dyon condensations in determining the various possible phases of gauge theories, including confinement, and demonstrating that other solitons, such as brane configurations, can play an important role in engineering the low-energy behaviour of theories. Moreover, in the presence of such configurations, the experimental upper bounds on the length of conjectured extra dimensions leapt upward by many orders of magnitude. Such a jump is unprecedented. Of course nature may not choose 


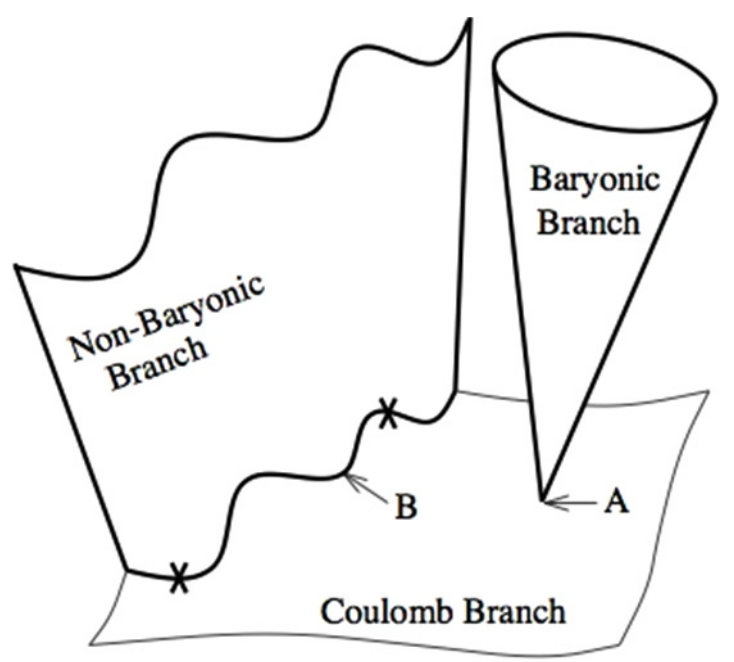

Figure 22. The theory has amazing properties: the ground states of the potentials are infinitely degenerate and are situated along a flat direction.

the possibility to posses sub-micron or so extra dimensions. It has also been found that dualities relating weak and small coupling, such as S duality, which were conjectured in some quantum field theories, are actually realized.

It was argued that, in the presence of gravity and blackholes, no global symmetries remain; string theory has made a much clearer case for that.

In quantum field theories, in particular those possessing some degree of supersymmetry, there were cases where the effective potential has flat directions. This feature became prominent in string theory. This led in many cases to the presence of many unwanted massless particles. Various sophisticated methods have been devised to get rid of them, with partial success. On the other hand, this behaviour highlighted the case of scale-invariant theories. In such quantum theories, the vacuum energy is independent of the scale generated by a spontaneous breaking of scale invariance. This takes the sting out of the original formulations of the cosmological constant problem. It also leads to considering a dilaton particle. The dilaton particle is also being reconsidered in the light of the special value of the mass of the Higgs particle (figures 22-23).

Present-day ideas such as inflation and a particle interpretation of what is called dark matter require extra particles. String theory provides (too) many of them (figure 24).

String theory has given support to the changes in paradigm that the presence of black holes forces upon us. In statistical mechanics, one is used to assuming that thermodynamical properties such as entropy are extensive. This applies for all energy scales. But at those energies at which black holes emerge, this breaks down. It was suggested that black hole entropies are proportional to the area of their horizon. This idea has been vindicated in string theory in the case when the metric is that of a constant negative curvature. Such spaces are called AdS spaces. In addition, it was found that ten-dimensional string theories in which space-time contains a five-dimensional $\mathrm{AdS}$ part are equivalent to

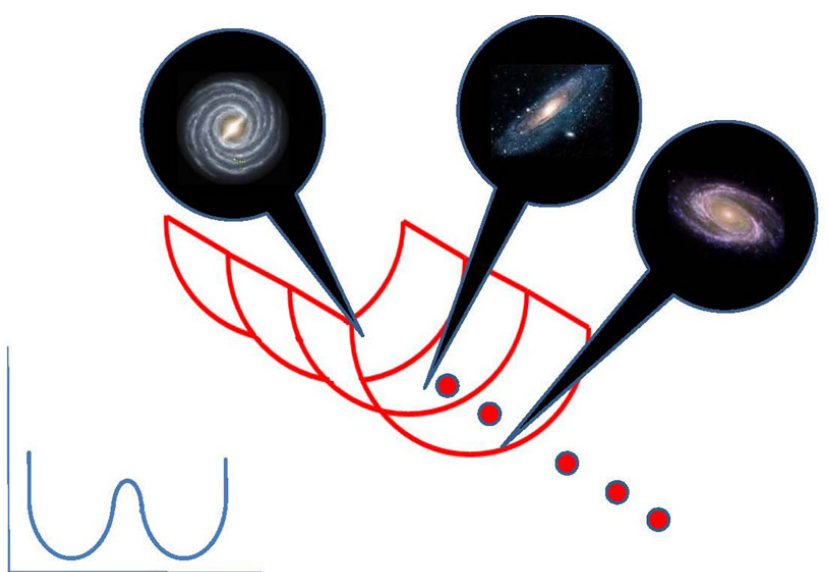

Figure 23. Illustration of a flat direction. The upper part shows the phase diagram of some theory. Each point corresponds to a different ground state. Each red point along this flat direction represents a different theory/universe. Credit: NASA (for the galaxies).

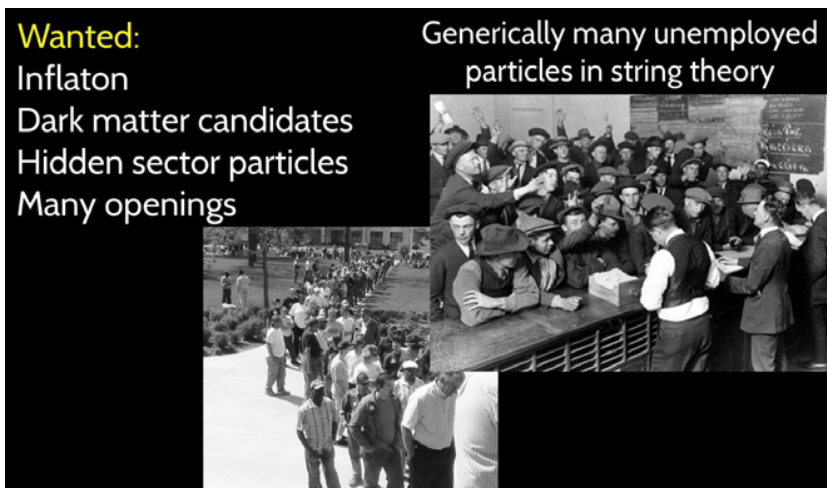

Figure 24. Theory needs many new particles, including those possibly related to inflation and the presense of dark matter. String theory has many light particles which beg to be used, hopefully some relation between the two can be found. Credit: (lower image) (C) Bernard Pollack. CC BY.
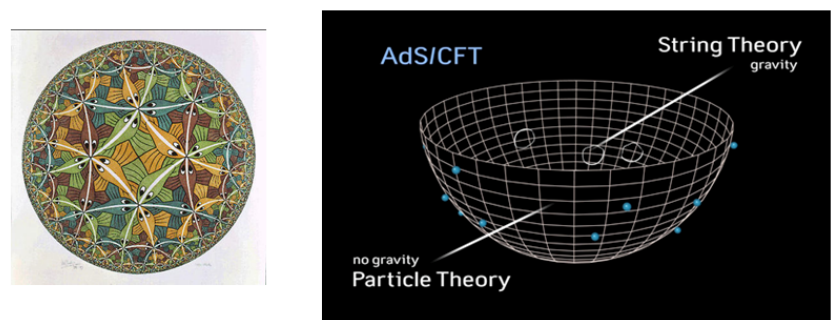

Figure 25. $N=4, d=4$, large $N$ SUSY YM SU( $N)$ is like a string theory on $\mathrm{AdS}_{5} \times \mathrm{S}^{5}$ with Flux $(2) 2013$ The MC Escher Company. The Netherlands. All rights reserved. Used with permission (www.mcescher.com).

special four-dimensional gauge theories which contain a high degree of supersymmetry, $N=4$ supersymmetry, but no gravity.

This vindicates the idea of holography and added to the magic of string theory in the process (figure 25).

A very intresting question is if field theory has in it all that is needed. The $N=4$ theory contains the whole cocktail composed of particles, strings, branes, and black holes of 


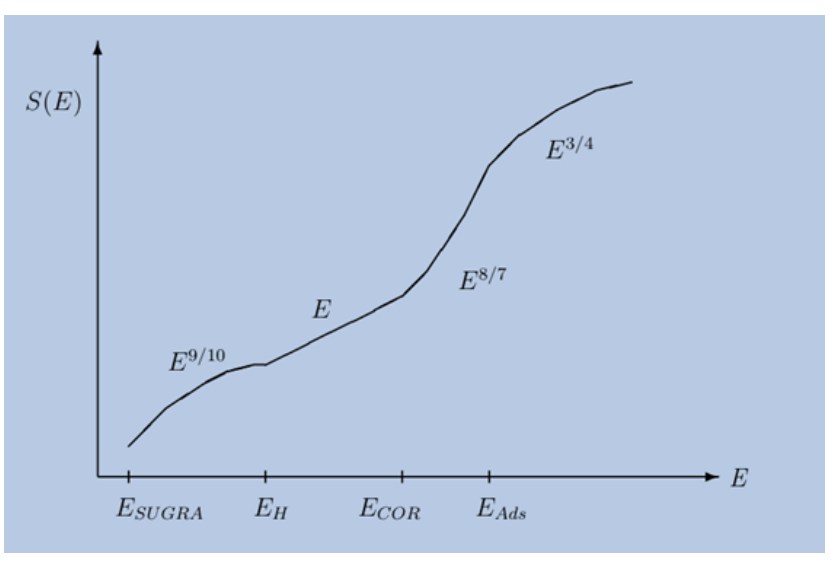

Figure 26. $N=4$ is best presented by different degrees of freedom at different energy ranges. From left to right, ten dimensioanl massless particles, strings, small blackholes in flat space and finally, at high energies, as black holes in AdS. The graph shows the energy dependence of the entropy of the system. Credit: among others J L F Barbon and E Rabinovici.

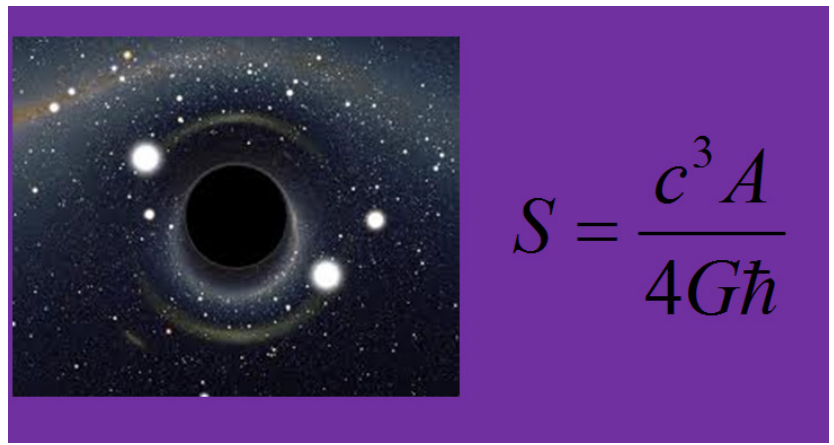

Figure 27. Black hole entropy is proportional to the area of its horizon. Credit: NASA.

all kinds. All are characters in one story. Each one has a range of energies in which it represents the relevant degrees of freedom; all phases of gravity manifested in a single system.

In particular, non-locality features of the bulk gravity are expressed by a totally different local system on the boundary of the bulk (figures 26 and 27).

This requires drastic changes in intuition. The entropy of the black hole is not proportional to its volume as we are used to at low energies, but only to the area of its horizon.

Moreover, the information of the black hole may be encoded on its horizon $\rightarrow$ HOLOGRAPHY

In addition, there have been various examples where string theory made it possible to calculate, microscopically, the entropy of certain black holes. It was vindicated that they obey the exact conjectured area law (figure 28).

The presence of black holes brought about several potential paradoxes. There is a body of evidence discovered in the AdS/CFT correspondence that there are setups where the full unitarity is present and the information paradox is absent (figure 29). Not all aspects of the issues are clear at this time, though.

In the process of punching holes in some of the arguments for a paradox, another question was answered en passant.
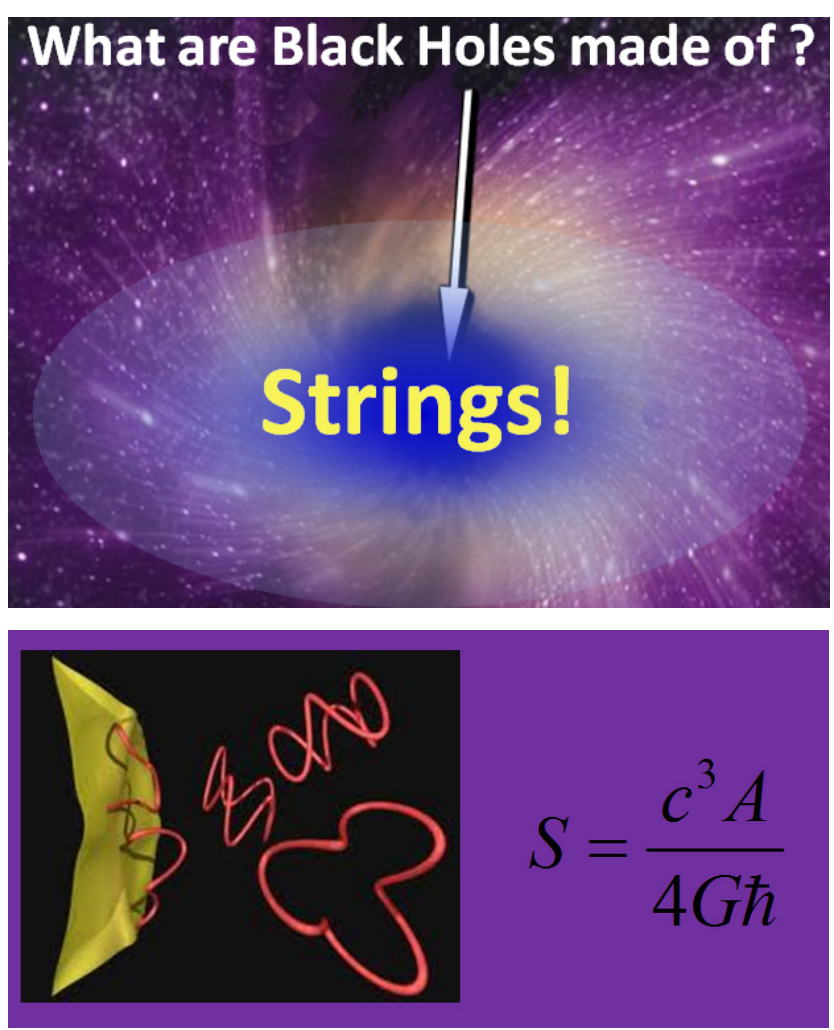

Figure 28. In some particular cases, the black hole entropy was obtained in terms of string theory microstates. Some amazing mathematical properites of the system were used to make the computation possible. Credit: (top) NASA.

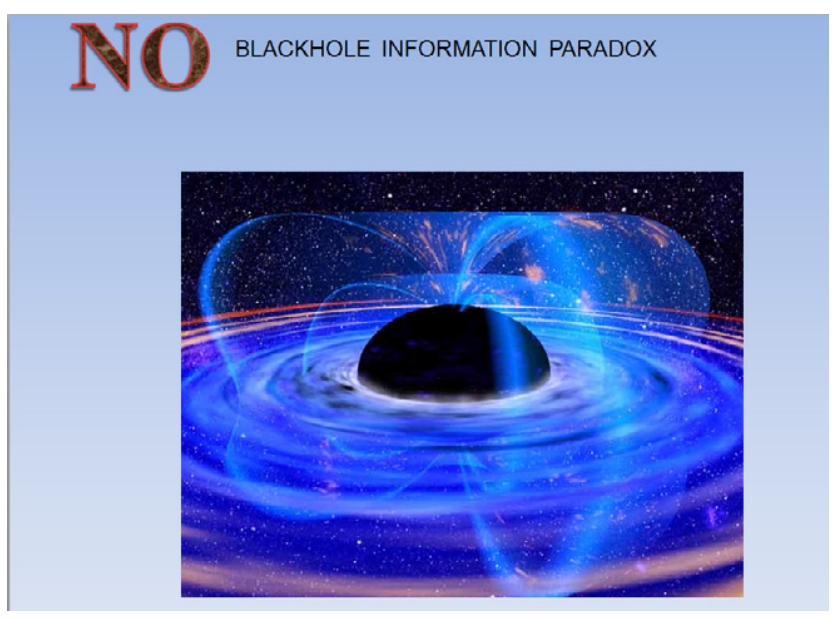

Figure 29. Holes have been punched in the original argument for a black hole information paradox. Credit: NASA.

Klein has shown that, in a relativistic interacting quantum field theory, the number of particles fails to be conserved and the different particle number sectors need to mix. In studying holography, it became clear that, in some cases, different topologies of space-time need to be considered; they also mix. All this assumes that holography is valid.

The issue of whether a given topology defines a super selection sector has been answered, the answer is no.

Different topologies need to mix for holography to work.

In this case thermal AdS mixes with black holes. They have different topologies (figure 30). 


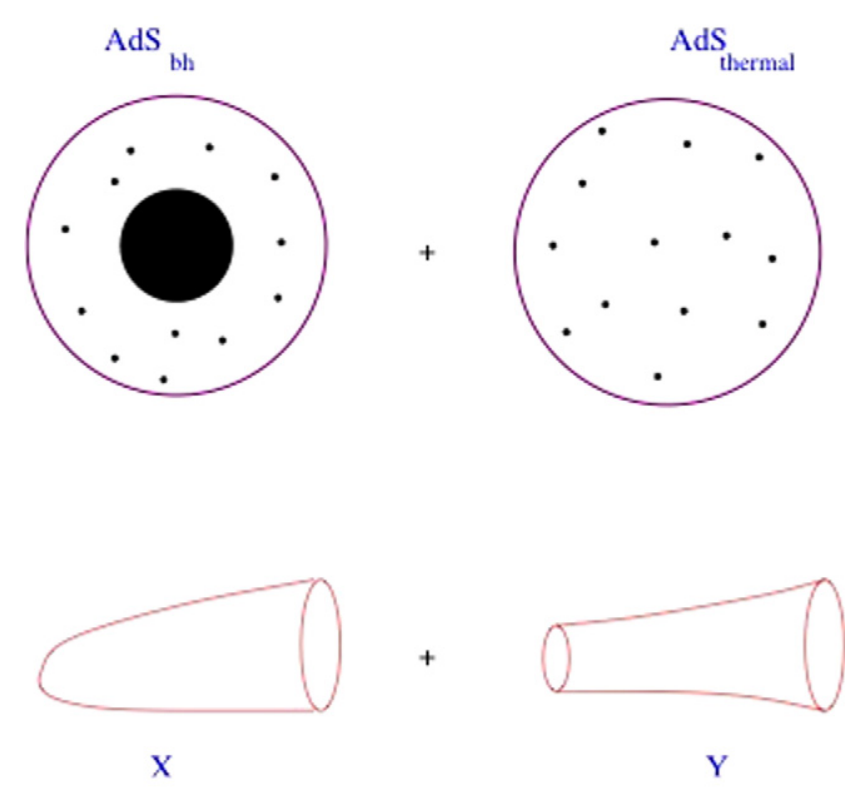

Figure 30. A thermal bath of particles in a thermal AdS background in the presence $(Y)$ or absence $(X)$ of a black hole. The backgrounds have different topologies, and yet both needed to be taken account of. Credit: J L F Barbon and E Rabinovici.

As stated before, there are also indications that, surprisingly, string theory can live with some singularities including some big crunches. One should recall that there is a new feature present in theories which are generally covariant. There are horizons behind which some unpleasant features can be hidden (figure 31).

Can one live with singularities? We claimed that there are examples where the singularities did not necessarily have to be a manifestation of the breakdown of our knowledge or approximation. The theory could live with them. String theory could-but does not need to-eventually heal them.

From its inception, string theory was born in the arena of hadronic phenomenology. The discovery that quantum chromodynamics as a field theory can very successfully describe the colour force between quarks has led to string theory retreating into a state of semi-hibernation. This period of near hibernation continues until 1984 when, thanks to the works of Green and Schwarz, interest in the field was rekindled. Invigorated, string theory tried not only to return to hadronic phenomenology, but to the Standard Model as a whole. At best, this attempt was only partially successful; it continues with various ups and downs to the beginning of the 21 st century.

But do string theory methods talk to actual experiments?

They definitely try! Theorists hunger to relate their work to data, however this is an all-important subject that deserves a talk of its own.

Returning to the statement, the significance of scientific research is measured not only by the quality of its results, but also by the quality of the questions it provokes, we note that many interesting questions did, indeed, come up. One was, what can fix the number of dimensions? Another is whether topologies can mix. And a yet more speculative question is
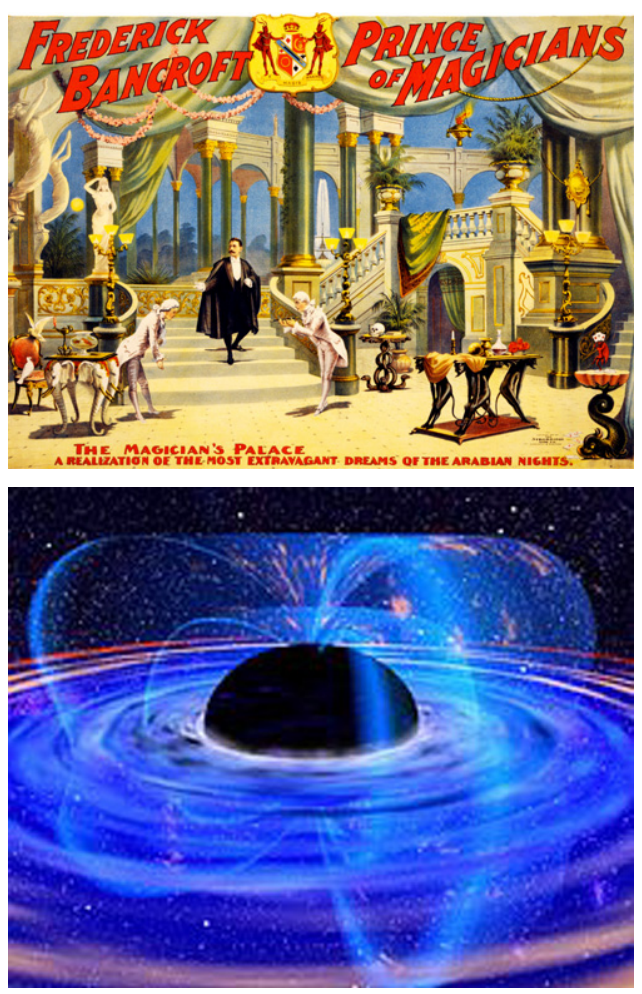

Figure 31. Gravity has magic cloaks behind which to hide its secrets. For example black hole horizons. Credit: (bottom) NASA.

whether there is only one universe to be considered. Ideas in eternal inflation, quantum gravity with its wheeler-DeWitt equation and string theory lead one to reconsider this question. Does the issue of the number of universes become a scientific question?

This is illustrated by a set of pictures in figure 32 . They show how the human horizon has expanded from the walls of a cave to a multiverse.

The idea of some form of a multiverse framework seems inevitable to some and too loose to be true to others. These issues do connect to the $\mathrm{LHC}$, but in a rather unexpected way.

Finally, at the LHC, what seems to be a fundamental scalar has been found. The mass of the particle was found to be in the range $125-126 \mathrm{GeV}$. It turns out that with this mass and very strong assumptions, in particular, that no new physics will emerge till a scale of about $10^{12} \mathrm{TeV}$, which is well below the Planck scale, our universe could be on the verge of being metastable (figures 33 and 34). It could well decay; it will take a very long time on a human scale but it could well happen (figure 35). In this case, our universe may search for another viable configuration. It is not very likely it will find it. This is only the tip of the iceberg....

There are many additional open issues left-let me list a few.

How are we to get rid of the constraints of the ambient space and the world-sheet, thereby finding a unified description for all phases of 'gravity'?

Do the laws of physics themselves and the interactions they contain also emerge? 

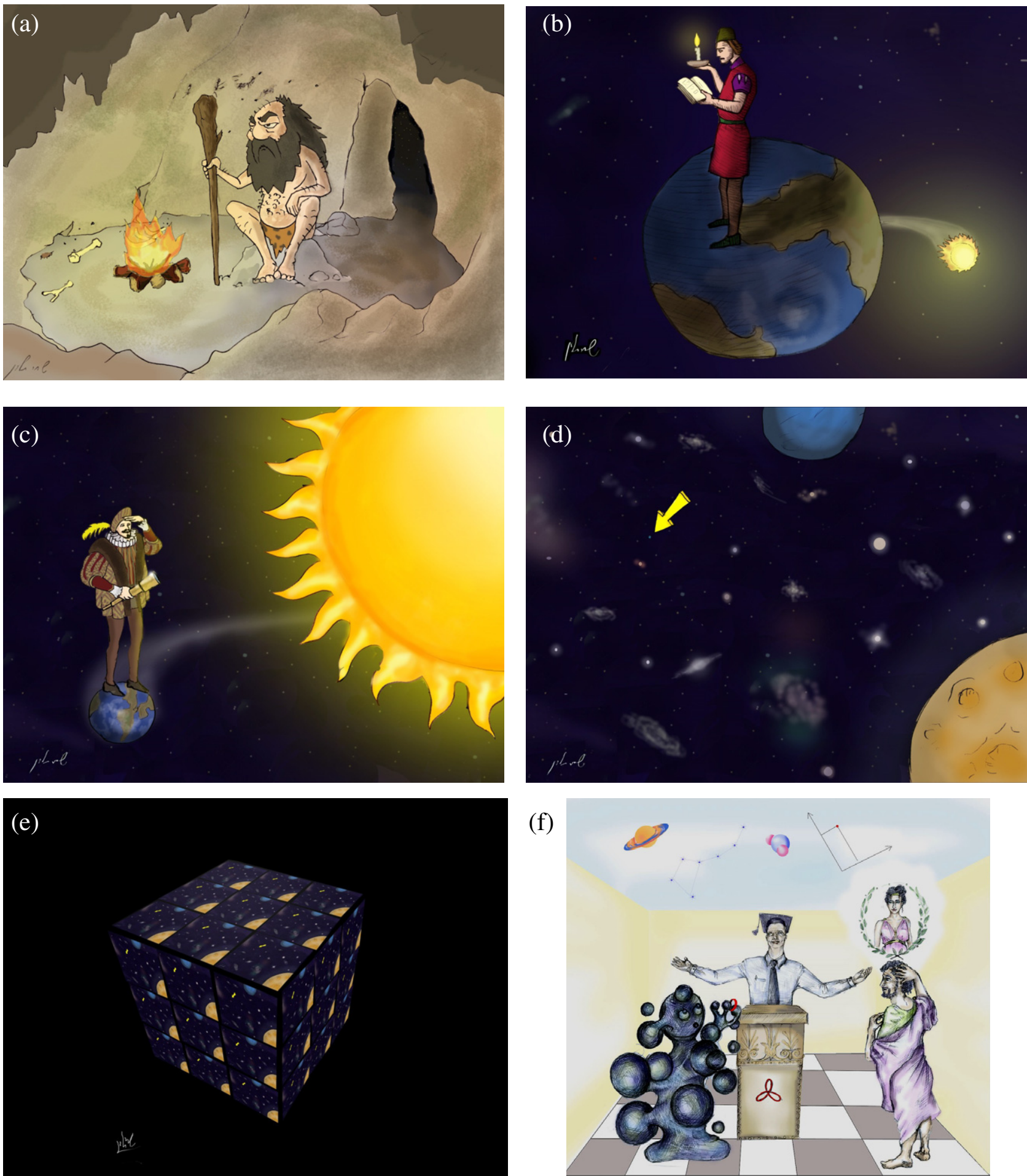

(f)

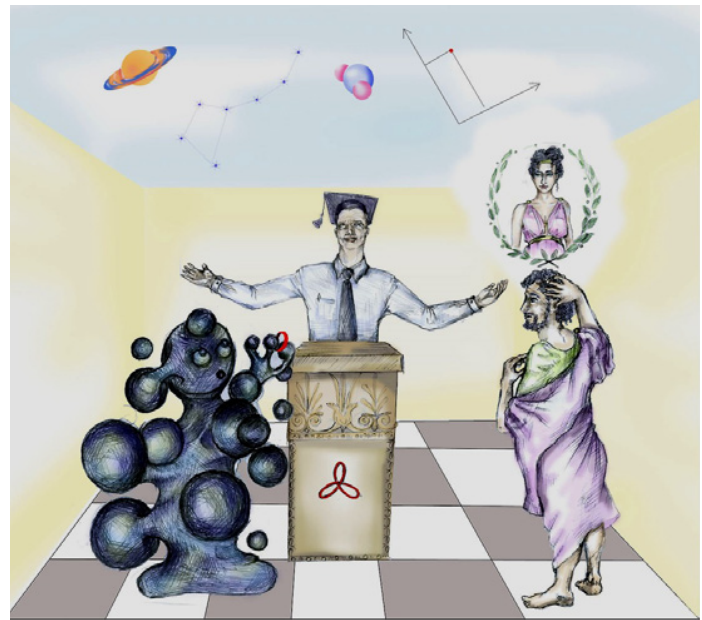

Figure 32. (a)-(e) A set of drawings showing the human perspective increasing from (a) the walls of a cave to (e) a multiverse. The last of these figures (f), describes a string/shotgun marriage between a confused scientist and the multiverse. Credits: (a)-(e) Shahar Tel Dan.

Is the role of $\alpha^{\prime}$ to provide a fundamental scale, or is the theory scale/conformal invariance such that a scale actually emerges only spontaneously?

One needs a much deeper understanding of time-dependent backgrounds such as cosmological ones.

There seems to be a vast amount of new structure waiting to be discovered behind the new higher-dimensional non-Lagrangian(?) quantum field theories in five and six dimensions.

What is the appropriate description for the current degrees of freedom to appear in different regimes as the relevant degrees of freedom?

Is there such a global description?

Will this impact on the description of nature? 

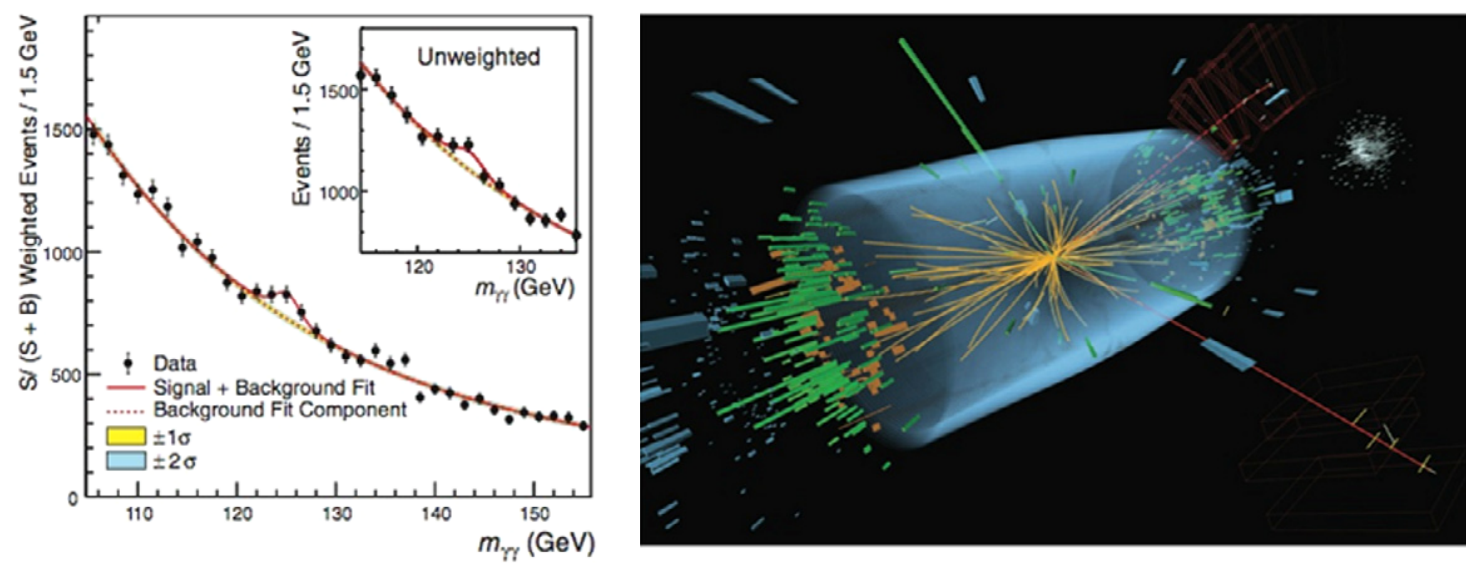

Figure 33. A new particle was discovered at CERN with a mass of $125-126 \mathrm{GeV}$. Credit: ATLAS Experiment () 2013 CERN.

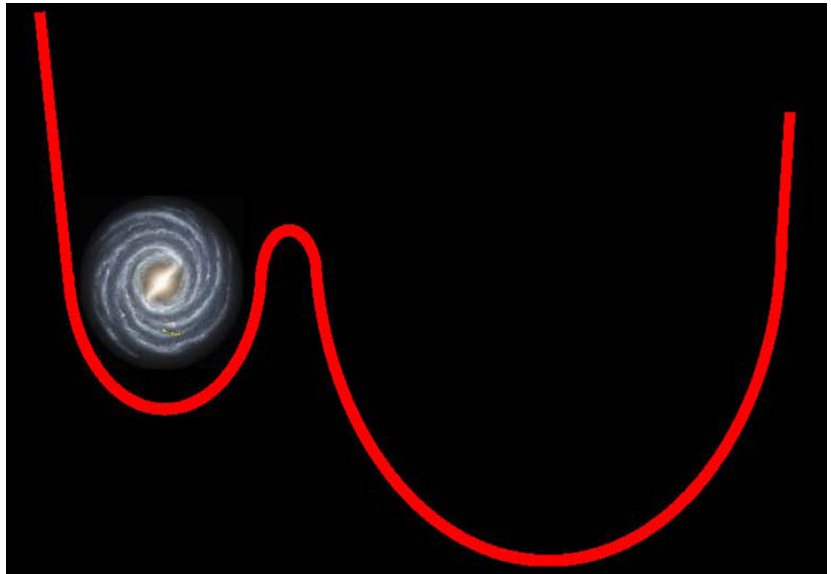

Figure 34. Under very strong assumptions, our universe may be in a meta-stable state, it may well be doomed to decay. The presence of a new stable minimum requires more assumptions. Credit: NASA/JPL-Caltech/R Hurt/SSC (for the photographic insert).

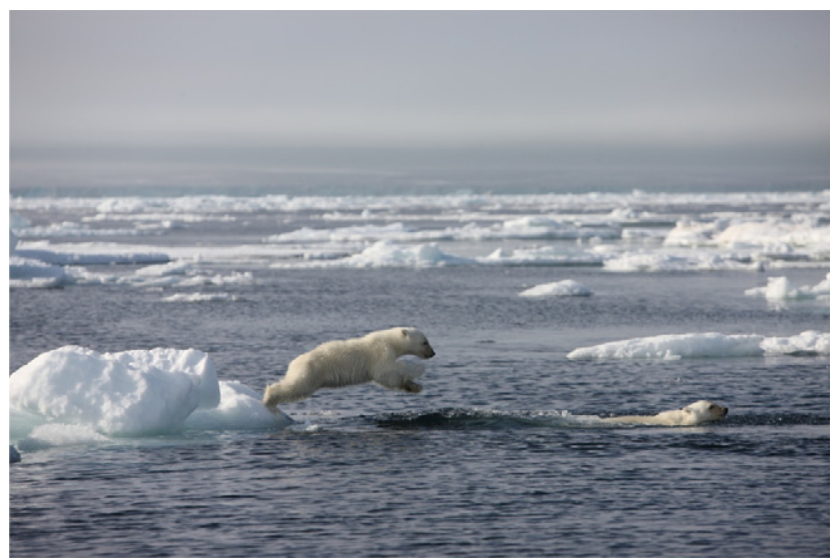

Figure 35. It is not easy to find a ground state in which life as we know it is possible. If our universe is only metastable, we will need to be lucky. Credit: Jason Roberts, Jason Roberts Productions.

We embarked on this journey seeking quests and I will end up with a thought on the quest for unification.

\section{Unification: beauty and doubts}

The idea of having one equation to determine all of the material world is tempting, but it also has a dark side. Should

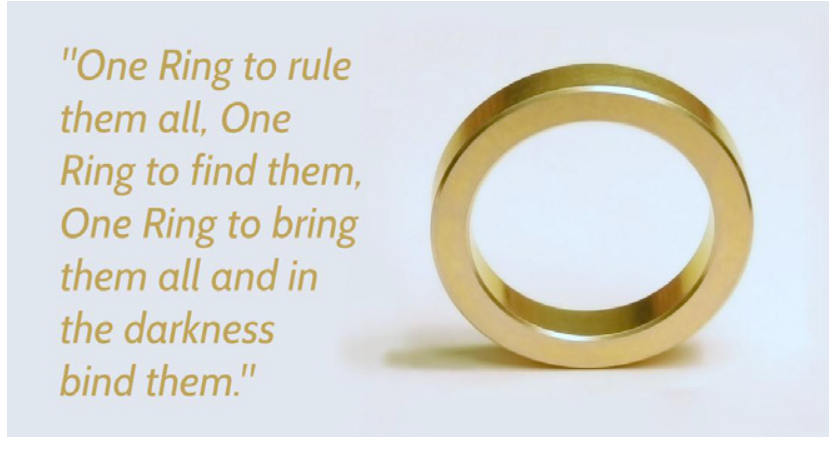

Figure 36. One equation to rule us all. Credit: quote reproduced by permission of Harper Collins Publishers Ltd, The Lord of the Rings (C) Fourth Age Limited 1954, 1955, 1966; image (C) Quazbut. CC BY.

one give so much authority to one single equation or set of equations (figure 36)?

How can one remove this concentrated power from the equation?

It seems an ingenious way was found: if the one equation has an infinite number of solutions, multi-universes if you wish, its power has been thoroughly diminished.

I will conclude with some thoughts which come up naturally from this discussion. We cannot avoid noticing that the more we strive for greater understanding and additional unification, the more we find our 'positioning' in the universe to be less central...

and then at the end we wonder why we have been privileged to realize this.

\section{Acknowledgments}

I would like to thank Professor Tord Ekelöf and the Nobel Foundation for organizing this very stimulating symposium. Many thanks to Shahar Tel Dan for his inspiring illustrations, and Ofer Arbeli, Roberto Auzzi, Jose Barbon, Tullio Basaglia, Sven Bjarke Gudnason and Ronen Ingbir for their invaluable help in preparing this presentation. I thank Jose Barbon for the many things I have learned from him, several of which appear in this presentation. I would like to express my gratitude to Jason Roberts of Jason Roberts Productions for kindly agreeing to let me reproduce figure 35 .

Apologies. Many thousands of researchers have contributed to the understanding of some properties of 
string theory. In this framework it is possible only to do injustice by singling out some works. I will thus refrain from giving references-including to my own work-in this volume and I trust the interested reader will be able to track the appropriate ones by herself.
The author has made best efforts to trace the copyright holders of all material reproduced in this article and apologises for any oversight in gaining relevant permissions. If any copyright material has not been acknowledged, please contact the author. 